\title{
High Nitrogen Fertilization of Tobacco Crop in Headwater Watershed Contaminates Subsurface and Well Waters with Nitrate
}

\author{
D. R. Kaiser, ${ }^{1}$ L. Sequinatto, ${ }^{2}$ D. J. Reinert, ${ }^{3}$ J. M. Reichert, ${ }^{3}$ \\ D. S. Rheinheimer, ${ }^{3}$ and L. Dalbianco ${ }^{3}$ \\ ${ }^{1}$ Federal University of South Frontier (UFFS), Avenida Major Antônio Cardoso 590, 97900-000 Cerro Largo, RS, Brazil \\ ${ }^{2}$ Soils and Natural Resources Department, State University of Santa Catarina (UDESC), Avenida Luis de Camões 2090, \\ 88520-000 Lages, SC, Brazil \\ ${ }^{3}$ Soils Department, Federal University of Santa Maria (UFSM), University Campus, 97106-900 Santa Maria, RS, Brazil \\ Correspondence should be addressed to J. M. Reichert; reichert@ufsm.br
}

Received 31 October 2014; Revised 27 December 2014; Accepted 20 January 2015

Academic Editor: Zongming Ren

Copyright (C) 2015 D. R. Kaiser et al. This is an open access article distributed under the Creative Commons Attribution License, which permits unrestricted use, distribution, and reproduction in any medium, provided the original work is properly cited.

Our hypothesis was that subsurface and well waters in watershed with shallow, stony soils, steep landscapes, and cropped to tobacco are contaminated by nitrate. Nitrate in soil solution was monitored in $(0.20 \mathrm{~m})$ and below $(0.5 \mathrm{~m})$ root zone with tension lysimeters, in five transects. Water from two wells (beneath tobacco field and in native forest) used for human consumption was also analyzed for nitrate. Soil bulk density, porosity, and saturated hydraulic conductivity were evaluated. Soil physical and hydrological properties showed great variation at different landscape positions and soil depths. Soil coarse grain size, high porosity, and saturated hydraulic conductivity favored leaching nitrate. Nitrate in soil solution from tobacco fields was greater than in natural environment. Nitrate reached depths bellow rooting zone with values as high as $80 \mathrm{mg} \mathrm{L}^{-1}$ in tobacco plantation. Water well located below tobacco plantation had high nitrate concentration, sometimes above the critical limit of $10 \mathrm{mg} \mathrm{L}^{-1}$. Tobacco cropping causes significant water pollution by nitrate, posing risk to human health. A large amount of nitrogen fertilizers applied to tobacco and nitrate in subsurface waters demonstrate the unsustainability of tobacco production in small farming units on steeps slopes, with stony and shallow soils.

\section{Introduction}

Studies in headwater watersheds are of noteworthy importance, since soil and water contamination at this scale, that is, zero-order watershed, affects water quality of higher-order watersheds and river basins. In small agricultural watersheds in Southern Brazil, pesticides [1, 2] and phosphorus are present in surface and well waters and sediments [3-7] in surface water in creeks. Although there exist management practices for soil and water protection [8], nitrate contamination in watersheds cropped to tobacco is a major concern.

Tobacco crops require large amounts of nitrogen until flowering, since deficiency during this period diminishes leaf yield and quality. Conversely, excess nitrogen in soil solution after tobacco flowering results in high leaf protein content, diminishing tobacco burning quality [9]. Nitrate regulates tobacco root system branching and allocation and distribution of carbohydrates between root system and aboveground biomass (leaves and stems) [10]. Thus, when there is low nitrate concentration in the rhizosphere, root growth and branching are stimulated rather than leaves and stems growth, which is an undesirable since tobacco leaves are the commercial component.

Tobacco companies strongly recommend that farmers apply high fertilizer doses, due to low nitrogen use efficiency by tobacco crop, especially in well-drained soils [11]. Nitrogen recovery rate diminishes as applied dose increases and rainfall increases (high precipitation) to compensate for nitrogen leaching and erosion loss [12].

Official recommendation foresees the use of complementary fertilization after frequent, intense rainfall [13]. This 


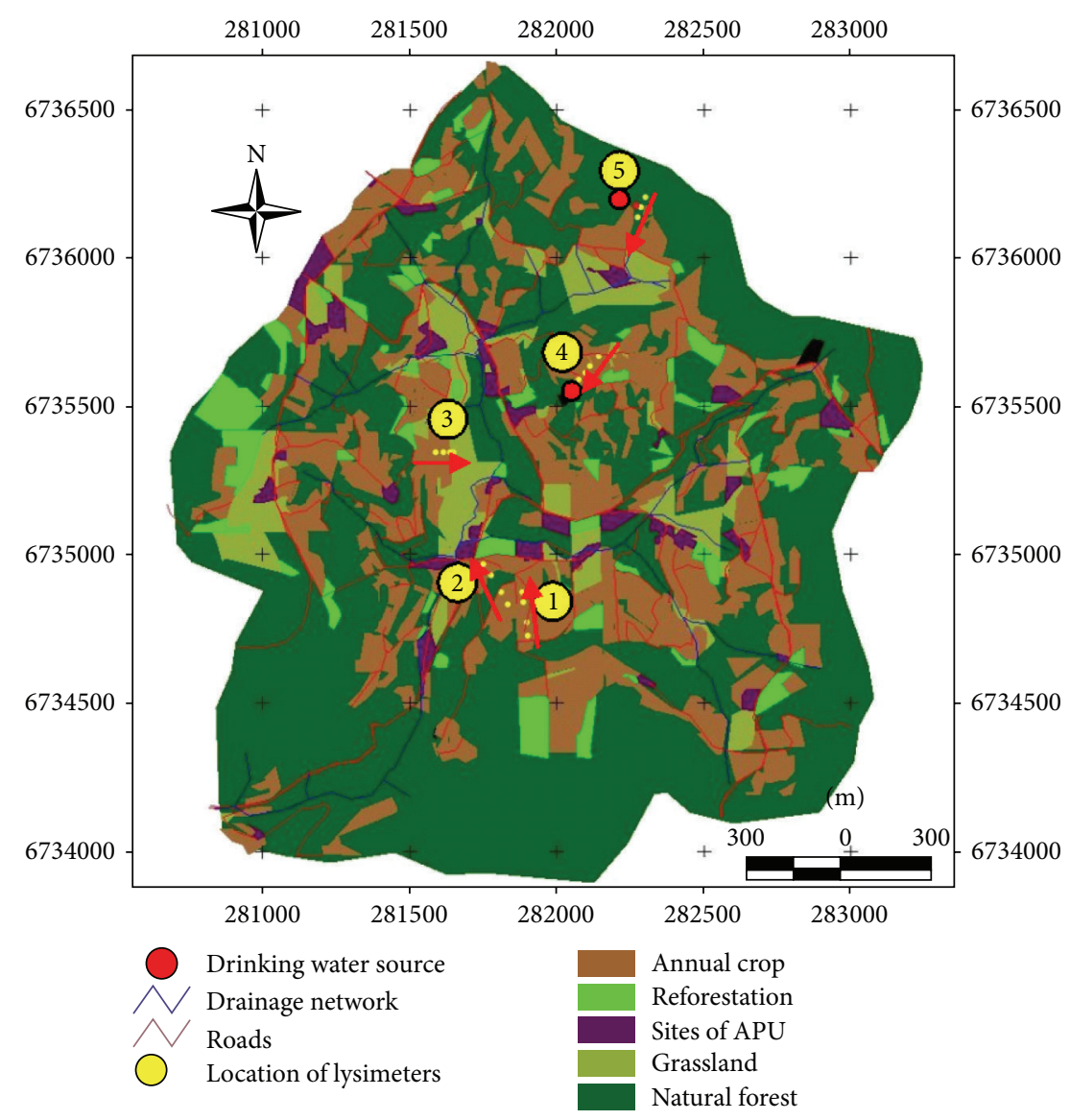

FIGURE 1: Collection sites on transects in the watershed. Points indicate transects in landscape and arrows indicate slope direction. APU: agricultural production units.

recommendation and especially the one adapted by tobacco companies require nitrogen to be applied in nitrate form. Although nitrate guarantees leaf quality [9], its high mobility in soil poses higher pollution potential.

Nitrate concentration in water (groundwater and surface water bodies) in natural environments is rarely more than $1 \mathrm{mg} \mathrm{L}^{-1}$ [14]. However, anthropic activities strongly change the nitrogen cycle, particularly by increasing nitrate in soil and in water. When nitrate concentration in water reaches values above $10 \mathrm{mg} \mathrm{L}^{-1}$, water is not recommended for human consumption [15], but values above $2 \mathrm{mg} \mathrm{L}^{-1}$ may already cause ecological imbalance in aquatic environments [14]. In Europe, nutrient doses are defined not only by crop requirements but also by contamination possibility, with a legal framework that farmers must follow to attain good longterm water quality [16].

Runoff and subsurface water flow enriches water with sediments and nutrients [17]. Soil texture, permeability, and water-storage capacity are main factors involved in water movement and determine groundwater contamination susceptibility $[18,19]$. On sloppy land and in shallow soils, infiltrated water may percolate among fissures of decomposing rocky material until it reaches groundwater. In low land regions, saturated zones and areas with source outcroppings are generally formed, and these constitute headwaters of many rivers and sites where water wells for human consumption are built. Thus, water quality is closely linked to land use and management, and it is an indicator of production systems impacts [20], especially in sites where soils have low waterstorage capacity [21].

Our hypothesis was that subsurface and well waters in tobacco fields and in downhill forest are contaminated by nitrate in shallow, stony soils on steep landscapes. Our objective was to evaluate nitrate concentration in soil solution of tobacco fields compared with native forest and grasslands, in water wells for human consumption, with native forest between well and tobacco field, and in native forest.

\section{Material and Methods}

2.1. Study Site. The study was done in Arroio Lino watershed, a small agricultural watershed (480 ha) in Southern Brazil (Figure 1). Climate is humid subtropical (Cfa type) according to Köppen classification. Maximum mean temperature is above $22^{\circ} \mathrm{C}$ and minimum temperature of the coldest month is between -3 and $18^{\circ} \mathrm{C}$. Rainfall is normally well distributed, ranging from 1300 to $1800 \mathrm{~mm}_{\text {year }}{ }^{-1}$, where May and June have more rainfall [16].

Landscape has steep slopes, occupied by entisols on slopes and mollisols on terraces [22]. Lands in the watershed are 
farmed by 36 families, whose main cash crop is tobacco leaves. During 2005/2006 harvest, 80.4 ha of tobacco was grown: 53.5 ha (67\%) under conventional tillage, 26 ha (32\%) with minimum tillage, and 0.8 ha (1\%) with no-till system. Soil is usually tilled with animal-drawn moldboard plough and disked with pin-harrow.

Fertilization practices are similar in all farming units, following tobacco-companies technological package, disconsidering soil analysis or agricultural history of the field. During 2005/2006 harvest cropping year, $36,900 \mathrm{~kg}$ of NPK (10-14-28) fertilizer was applied at transplanting tobacco seedlings and $6,850 \mathrm{~kg}$ of urea (45-00-00) plus $51,400 \mathrm{~kg}$ of Chile saltpeter (14-00-14) as topdressing fertilization on the 80.4 ha of tobacco crop.

During soil tillage, existing cover crop is plowed under with a moldboard plow and seedbed rows are prepared. Tobacco is planted in ridged rows spaced $1.2 \mathrm{~m}$ apart, plants are spaced $0.5 \mathrm{~m}$ apart in the row, and fertilizer is applied manually. Topdressing fertilization is usually divided into two applications: the first 20 days after transplanting is done by laterally distributing either urea or Chile saltpeter on the row and incorporating it into soil by moldboard plowing or applying it next to seedlings with a handheld fertilizer spreader and the second 40 days after transplantation is done with handheld fertilizer spreader for Chile saltpeter.

2.2. Experiment Design. Five transects were strategically chosen in the landscape, including tobacco fields, native forests, and grasslands (Figure 2). In a control transect, we chose three points situated on native forest in different landscape altitudes. For the other four transects with tobacco crops, four points were chosen: in transect 1 , all points were in tobacco fields; in transect 2 , two points were in tobacco fields (highland) and two in grassland (lowland); in transect 3, tobacco crops area is separated by a strip of native forest, where three points were chosen in tobacco fields (two above and one below native forest) and one point was chosen in native forest; and, in transect 4 , the lowest point was in native forest and three other points were in tobacco fields. Landscape positions along the transects were 1, upper slope, 2, mid slope, 3, lower slope, and 4, plateau. Automatic tensiometers to measure the matric potential of soil water were installed, but they were inefficient because of the stony soil and, thus, it was not possible to estimate the water flow in the soil.

Water from two wells was sampled to determine quality of water used for human consumption. The first well was in the control transect and the second well on lowest part of transect 4. Although the well in transect 4 was positioned in lowlands, there was a strip of native forest between the well and tobacco crop field.

2.3. Sampling and Sample Analysis. Nitrate concentration in tobacco-fields soil solution was monitored in and below root growth zone. Water was collected by tension (suction) lysimeters with porous ceramic cups [23], installed at 0.2 and $0.5 \mathrm{~m}$ depth in tobacco rows. Porous cup lysimeters are considered an effective methodology to collect and evaluate soil solution [24-27]. In total, there were 40 monitoring points in the watershed: 24 tension lysimeters installed in tobacco fields, 10 in native forest, and 6 in pasture. The number of collection points was proportional to the land use in the watershed. The collection of soil solution occurred after 60 days of transplanting (DAT) tobacco seedlings, which is the period after the last topdressing with Chile saltpeter. The dates of the collections were period $1=$ November 10, 2005 (60 DAT); period 2 = November 28, 2005 (78 DAT); period 3 = December 9, 2005 (89 DAT); period $4=$ January 6, 2006 (117 DAT); period 5 = January 20, 2006 (131 DAT); period 6 = January 27, 2006 (138 DAT); and period 7 = February 10, 2006 (145 DAT).

Soil samples were collected at four depths (0.0-0.1, 0.1$0.2,0.2-0.3$, and $0.3-0.4 \mathrm{~m}$ ) with metal rings $(0.04 \mathrm{~m}$ high and $0.06 \mathrm{~m}$ diameter) to determine soil bulk density, porosity, saturated hydraulic conductivity, and particle-size distribution. In forested areas, soil samples were not collected because of excessive amount of roots and stony soil surface.

To extract soil solution, $50 \mathrm{kPa}$ tension was applied to lysimeters with a manual vacuum pump immediately after rainfall events. Soil solution extracted by lysimeters was collected three days after tension application, and samples were stored in $50 \mathrm{~mL}$ glass vials previously washed with cleaning solution $\left(0.0125 \mathrm{~mol} \mathrm{~L}^{-1} \mathrm{HCl}\right)$ and dried in a laboratory oven at $105^{\circ} \mathrm{C}$ for $24 \mathrm{~h}$. Between samplings, vacuum pump tubes were cleaned with cleaning solution and distilled water to avoid contamination of soil solution samples. Vials containing soil solution samples were stored in polystyrene boxes and maintained under refrigeration until laboratory analysis. Nitrate content was determined by the distillation method with a semi-micro-Kjeldahl procedure [28].

2.4. Data Analyses. Statistical analysis for physical properties and nitrate concentrations in soil solution, evaluated in different positions and soil uses, consisted of orthogonal contrasts using the SAS [29]. The contrasts were set up between different soil use conditions (tobacco $\times$ grassland; tobacco $\times$ forest), landscape positions (upper slope $\times$ mid slope; upper slope $\times$ lower slope; upper slope $\times$ plateau), and time of soil solution collection $(60 \mathrm{DAT} \times 78 \mathrm{DAT}, 60 \mathrm{DAT}$ $\times 89 \mathrm{DAT}, 60 \mathrm{DAT} \times 117 \mathrm{DAT}, 60 \mathrm{DAT} \times 131 \mathrm{DAT}, 60 \mathrm{DAT} \times$ 138 DAT, and 60 DAT $\times 145$ DAT).

\section{Results}

3.1. Soil Physical and Hydraulic Characterization. Soils at collection sites are pedogenetically young and have large amount of coarse fractions (gravel and pebbles) (Table 1). Clay contents are higher in lower and flatter landscape positions, where soils are more developed and less eroded and are with sediment deposition.

Soils have low bulk density and high total soil porosity, macroporosity, and saturated hydraulic conductivity (Table 2). Because of conventional soil tillage, surface soil layer has significantly lower density and higher porosity when compared to native fields. Mean values of soil bulk density, total porosity, macroporosity, and microporosity 

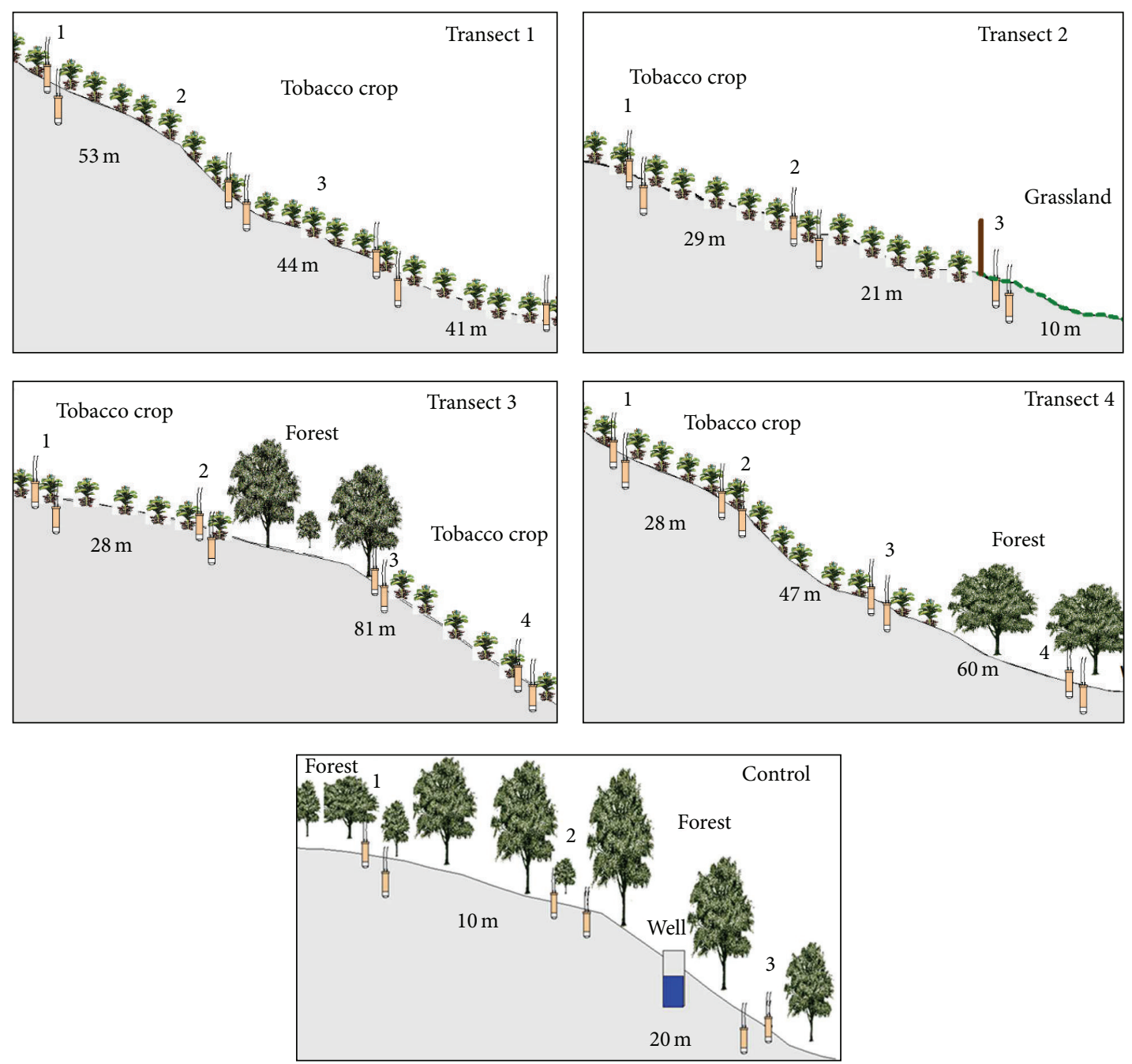

FIGURE 2: Distribution of collection points for transects with different soil uses. Points indicate lysimeters in transects along the landscape.

were, respectively, $1.18 \mathrm{Mg} \mathrm{m}^{-3}, 0.56 \mathrm{~m}^{3} \mathrm{~m}^{-3}, 0.20 \mathrm{~m}^{3} \mathrm{~m}^{-3}$, and $0.36 \mathrm{~m}^{3} \mathrm{~m}^{-3}$. These conditions are favorable to soil water flow and may contribute to increased nitrate leaching. Saturated hydraulic conductivity (Ks) varied greatly among soil layers and collection sites from different landscape positions (from 47 to $609 \mathrm{~mm} \mathrm{~h}^{-1}$ and $298 \mathrm{~mm} \mathrm{~h}^{-1}$ the average value; Table 2). These values are considered high [30], mainly because of the coarse granulometry [31,32], and indicate that under saturated soil conditions water flow is fast and favors groundwater contamination.

3.2. Rainfall. Rainfall was frequent and well distributed during the tobacco crop cycle (Figure 3). During the study period, total rainfall was $1079 \mathrm{~mm}$, but in the first $380 \mathrm{~mm}$ of rain, nitrate losses were not monitored.

3.3. Nitrate Contents in Soil Solution from Natural Environment. Nitrate concentration in soil solution from native environment (grassland and forest) was low when compared

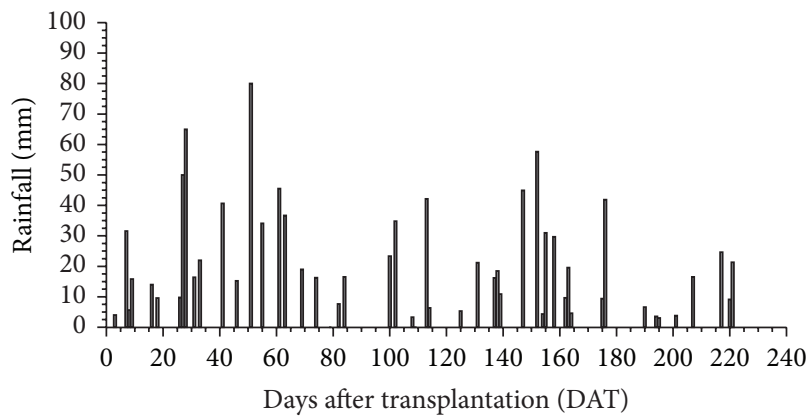

Figure 3: Rainfall during tobacco crop cycle.

to values found within tobacco crops, even if organic matter contents are higher (Table 3 ).

3.4. Nitrate Contents in Soil Solution from Tobacco Crops. Nitrate concentration in tobacco crops soil solution varied with landscape position, depth, and period of collection. 
TABLE 1: Particle-size distribution in soil surface layer at sampling points, for five transects and different points in the watershed.

\begin{tabular}{|c|c|c|c|c|c|c|c|}
\hline Transect & Position/use & Soil layer (m) & Pebbles & Gravel & $\begin{array}{r}\text { Sand } \\
\mathrm{g} \mathrm{kg}^{-1} \\
\end{array}$ & Silt & Clay \\
\hline \multirow{2}{*}{1} & \multirow{2}{*}{1, tobacco } & $0.0-0.2$ & 470 & 87 & 177 & 196 & 70 \\
\hline & & $0.2-0.4$ & 292 & 190 & 152 & 247 & 119 \\
\hline \multirow{2}{*}{1} & \multirow{2}{*}{2, tobacco } & $0.0-0.2$ & 272 & 91 & 259 & 292 & 85 \\
\hline & & $0.2-0.4$ & 317 & 58 & 270 & 261 & 95 \\
\hline \multirow{2}{*}{1} & \multirow{2}{*}{3 , tobacco } & $0.0-0.2$ & 299 & 20 & 313 & 259 & 109 \\
\hline & & $0.2-0.4$ & 336 & 96 & 247 & 224 & 97 \\
\hline \multirow{2}{*}{1} & \multirow{2}{*}{4 , tobacco } & $0.0-0.2$ & 241 & 86 & 260 & 309 & 103 \\
\hline & & $0.2-0.4$ & 543 & 0 & 186 & 203 & 68 \\
\hline \multirow{2}{*}{2} & \multirow{2}{*}{1, tobacco } & $0.0-0.2$ & 152 & 0 & 308 & 314 & 226 \\
\hline & & $0.2-0.4$ & 141 & 0 & 279 & 230 & 350 \\
\hline \multirow{2}{*}{2} & \multirow{2}{*}{2, tobacco } & $0.0-0.2$ & 119 & 0 & 415 & 292 & 175 \\
\hline & & $0.2-0.4$ & 143 & 0 & 345 & 316 & 196 \\
\hline 2 & 3 , forest & $0.0-0.2$ & 383 & 251 & 164 & 149 & 55 \\
\hline \multirow{2}{*}{3} & \multirow{2}{*}{1, tobacco } & $0.0-0.2$ & 148 & 100 & 313 & 360 & 81 \\
\hline & & $0.2-0.4$ & 437 & 90 & 238 & 169 & 65 \\
\hline \multirow{2}{*}{3} & \multirow{2}{*}{2, tobacco } & $0.0-0.2$ & 204 & 65 & 314 & 315 & 102 \\
\hline & & $0.2-0.4$ & 51 & 0 & 358 & 434 & 156 \\
\hline \multirow{2}{*}{3} & \multirow{2}{*}{ 3, grassland } & $0.0-0.2$ & 122 & 86 & 404 & 297 & 90 \\
\hline & & $0.2-0.4$ & 75 & 0 & 453 & 358 & 114 \\
\hline 3 & 4, grassland & $0.0-0.2$ & 151 & 273 & 294 & 223 & 59 \\
\hline \multirow{2}{*}{4} & \multirow{2}{*}{1 , tobacco } & $0.0-0.2$ & 294 & 0 & 397 & 187 & 122 \\
\hline & & $0.2-0.4$ & 151 & 231 & 352 & 156 & 109 \\
\hline \multirow{2}{*}{4} & \multirow{2}{*}{2, tobacco } & $0.0-0.2$ & 249 & 101 & 362 & 183 & 104 \\
\hline & & $0.2-0.4$ & 156 & 130 & 362 & 212 & 139 \\
\hline \multirow{2}{*}{4} & \multirow{2}{*}{3 , tobacco } & $0.0-0.2$ & 253 & 395 & 121 & 146 & 83 \\
\hline & & $0.2-0.4$ & 176 & 302 & 220 & 191 & 110 \\
\hline
\end{tabular}

Pebbles (>20 mm), gravel $(2-20 \mathrm{~mm})$, sand $(2-0.05 \mathrm{~mm})$, silt $(0.05-0.002 \mathrm{~mm})$, and clay $(<0.002 \mathrm{~mm}) .{ }^{*}$ Position: 1 : upper slope, 2 : mid slope, 3 : lower slope, and 4: plateau.

The flatter areas in the landscape (Table 3, point 4) had higher nitrate concentration in soil solution (Table 4) when compared to higher and steeper positions in the landscape (Table 3, point 1).

Tobacco crops presented the largest nitrate contents predominantly from applied nitrogen fertilizers. When comparing different depths $(0.20$ and $0.50 \mathrm{~m})$, nitrate concentrations were similar, although these concentrations tended to decrease over time, with the highest value immediately after fertilization.

3.5. Nitrate Contents from Sources Used for Human Consumption. Nitrate concentration in water from domestic wells varied over the monitored period (Figure 4). The water source located in native forest without neighboring tobacco crops (control well) presented the lowest nitrate concentration (Table $3 ; 2.6 \mathrm{mg} \mathrm{L}^{-1}$ of $\mathrm{N}^{-\mathrm{NO}_{3}}{ }^{-}$), whereas in water well nearby a tobacco field in higher position nitrate concentration reached critical levels, varying from 8.5 to $12.8 \mathrm{mg} \mathrm{L}^{-1}$, almost always considered improper for human consumption in four different collection dates.

\section{Discussion}

Soil heterogeneity [33] and coarse granulometry [30] induced great variability in soil saturated hydraulic conductivity (Ks) among different monitored landscape positions and soil layers (Table 2). High soil porosity and saturated hydraulic conductivity benefit water infiltration, creating an environment favorable to solute transport into groundwater. Furthermore, soil surface is very stony, contributing to runoff reduction and water infiltration increase $[30,34]$.

In natural environments, plant biomass and soil organic matter store large amounts of organic nitrogen. However, nitrate concentrations in soil solution from natural environments (grassland and forest) are low, below $2 \mathrm{mg} \mathrm{L}^{-1}$ of $\mathrm{N}$ $\mathrm{NO}_{3}{ }^{-}$[35], mainly due to synchronicity between nitrogen mineralization and plant absorption or immobilization by microorganisms.

Ecosystem dynamics for nitrogen is extremely complex. Slash-and-burn of native vegetation frequently used in tobacco crops leads to rapid decline in soil organic matter (low resistance). During fallow period, fast revegetation occurs because of high nutrient availability, including 
TABLE 2: Bulk density (Bd), total porosity (Tp), macroporosity (Ma), microporosity (Mi), and saturated hydraulic conductivity (Ks) of soil for five transects and different points in the watershed.

\begin{tabular}{|c|c|c|c|c|c|c|c|}
\hline Transect & Position/use ${ }^{* *}$ & Soil layer (m) & $\begin{array}{c}\mathrm{Bd} \\
\mathrm{Mg} \mathrm{m}^{-3}\end{array}$ & $\mathrm{Tp}$ & $\begin{array}{c}\mathrm{Ma} \\
\mathrm{m}^{3} \mathrm{~m}^{-3}\end{array}$ & $\mathrm{Mi}$ & $\begin{array}{c}\mathrm{Ks} \\
\mathrm{mm} \mathrm{h}^{-1}\end{array}$ \\
\hline \multirow{4}{*}{1} & \multirow{4}{*}{1 , tobacco } & $0.0-0.1$ & 1.01 & 0.65 & 0.33 & 0.32 & 482 \\
\hline & & $0.1-0.2$ & 1.04 & 0.60 & 0.27 & 0.33 & 201 \\
\hline & & $0.2-0.3$ & 0.92 & 0.59 & 0.30 & 0.29 & 209 \\
\hline & & $0.3-0.4$ & 1.23 & 0.57 & 0.19 & 0.38 & $-^{*}$ \\
\hline \multirow{4}{*}{1} & \multirow{4}{*}{2 , tobacco } & $0.0-0.1$ & 1.11 & 0.38 & 0.13 & 0.25 & 316 \\
\hline & & $0.1-0.2$ & 1.23 & 0.56 & 0.19 & 0.36 & 461 \\
\hline & & $0.2-0.3$ & 1.15 & 0.58 & 0.21 & 0.36 & 183 \\
\hline & & $0.3-0.4$ & 1.19 & 0.56 & 0.20 & 0.36 & 148 \\
\hline \multirow{3}{*}{1} & \multirow{3}{*}{3 , tobacco } & $0.0-0.1$ & 1.06 & 0.61 & 0.28 & 0.33 & 429 \\
\hline & & $0.1-0.2$ & 1.19 & 0.58 & 0.22 & 0.36 & 449 \\
\hline & & $0.2-0.3$ & 1.16 & 0.61 & 0.21 & 0.40 & 171 \\
\hline \multirow{3}{*}{1} & \multirow{3}{*}{4 , tobacco } & $0.0-0.1$ & 1.22 & 0.57 & 0.14 & 0.43 & 151 \\
\hline & & $0.1-0.2$ & 1.16 & 0.57 & 0.14 & 0.42 & 94 \\
\hline & & $0.2-0.3$ & 1.07 & 0.57 & 0.15 & 0.42 & 315 \\
\hline \multirow{4}{*}{2} & \multirow{4}{*}{1, tobacco } & $0.0-0.1$ & 0.97 & 0.64 & 0.33 & 0.31 & 375 \\
\hline & & $0.1-0.2$ & 1.33 & 0.58 & 0.25 & 0.34 & 138 \\
\hline & & $0.2-0.3$ & 1.27 & 0.53 & 0.12 & 0.41 & 137 \\
\hline & & $0.3-0.4$ & 1.14 & 0.65 & 0.09 & 0.56 & 554 \\
\hline \multirow{3}{*}{2} & \multirow{3}{*}{2 , tobacco } & $0.0-0.1$ & 1.32 & 0.53 & 0.12 & 0.41 & 201 \\
\hline & & $0.1-0.2$ & 1.26 & 0.53 & 0.15 & 0.38 & 215 \\
\hline & & $0.2-0.3$ & 1.11 & 0.55 & 0.21 & 0.34 & 345 \\
\hline \multirow{4}{*}{2} & \multirow{4}{*}{3 , forest } & $0.0-0.1$ & 1.02 & 0.53 & 0.29 & 0.24 & 609 \\
\hline & & $0.1-0.2$ & 1.08 & 0.55 & 0.26 & 0.29 & 510 \\
\hline & & $0.2-0.3$ & 1.11 & 0.54 & 0.26 & 0.29 & 564 \\
\hline & & $0.3-0.4$ & 1.12 & 0.56 & 0.27 & 0.28 & $-^{*}$ \\
\hline \multirow{4}{*}{3} & \multirow{4}{*}{1 , forest } & $0.0-0.1$ & 1.14 & 0.58 & 0.17 & 0.42 & 138 \\
\hline & & $0.1-0.2$ & 1.10 & 0.59 & 0.20 & 0.39 & 130 \\
\hline & & $0.2-0.3$ & 1.31 & 0.56 & 0.11 & 0.45 & 104 \\
\hline & & $0.3-0.4$ & 1.15 & 0.59 & 0.17 & 0.41 & 362 \\
\hline \multirow{4}{*}{3} & \multirow{4}{*}{2 , forest } & $0.0-0.1$ & 1.06 & 0.63 & 0.26 & 0.37 & 321 \\
\hline & & $0.1-0.2$ & 1.16 & 0.61 & 0.19 & 0.42 & 103 \\
\hline & & $0.2-0.3$ & 1.26 & 0.54 & 0.10 & 0.44 & 197 \\
\hline & & $0.3-0.4$ & 1.10 & 0.63 & 0.17 & 0.46 & 378 \\
\hline \multirow{4}{*}{3} & & $0.0-0.1$ & 1.31 & 0.54 & 0.11 & 0.42 & 113 \\
\hline & 3 oracsland & $0.1-0.2$ & 1.31 & 0.57 & 0.16 & 0.41 & 95 \\
\hline & 3 , grassland & $0.2-0.3$ & 1.26 & 0.55 & 0.14 & 0.41 & 54 \\
\hline & & $0.3-0.4$ & 1.42 & 0.50 & 0.12 & 0.38 & 112 \\
\hline & & $0.0-0.1$ & 1.21 & 0.56 & 0.09 & 0.47 & 176 \\
\hline 3 & 4, grassland & $0.1-0.2$ & 1.35 & 0.53 & 0.16 & 0.37 & 336 \\
\hline & & $0.2-0.3$ & 1.34 & 0.53 & 0.15 & 0.38 & 47 \\
\hline & & $0.0-0.1$ & 1.19 & 0.55 & 0.25 & 0.30 & 585 \\
\hline & & $0.1-0.2$ & 1.08 & 0.55 & 0.26 & 0.29 & 463 \\
\hline 4 & 1 , tobacco & $0.2-0.3$ & 1.11 & 0.54 & 0.26 & 0.29 & 452 \\
\hline & & $0.3-0.4$ & 1.12 & 0.56 & 0.27 & 0.28 & 451 \\
\hline & & $0.0-0.1$ & 1.11 & 0.52 & 0.27 & 0.26 & 374 \\
\hline 4 & 2 , tobacco & $0.1-0.2$ & 1.18 & 0.51 & 0.27 & 0.25 & 490 \\
\hline & & $0.2-0.3$ & 1.24 & 0.52 & 0.24 & 0.28 & 389 \\
\hline & & $0.0-0.1$ & 1.16 & 0.53 & 0.26 & 0.27 & 447 \\
\hline 4 & & $0.1-0.2$ & 1.30 & 0.50 & 0.19 & 0.31 & 142 \\
\hline 4 & 3 , tobacco & $0.2-0.3$ & 1.23 & 0.51 & 0.24 & 0.27 & 427 \\
\hline & & $0.3-0.4$ & 1.45 & 0.43 & 0.14 & 0.28 & 435 \\
\hline Mean & & & 1.18 & 0.56 & 0.20 & 0.36 & 298 \\
\hline
\end{tabular}

${ }^{*}$ Not possible to measure due to very coarse texture. ${ }^{* *}$ Points: 1: upper slope, 2: mid slope, 3: lower slope, and 4: plateau. 
TABLE 3: Nitrate concentration in soil solution under different conditions of land uses and landscape positions in the watershed.

\begin{tabular}{|c|c|c|c|c|c|c|c|c|c|c|}
\hline \multirow{3}{*}{ Transect } & \multirow{3}{*}{ Position/use } & \multirow{3}{*}{ Depth (m) } & \multicolumn{8}{|c|}{ Date of collection } \\
\hline & & & $\begin{array}{c}\text { November } \\
10,2005\end{array}$ & $\begin{array}{c}\text { November } \\
28,2005\end{array}$ & $\begin{array}{c}\text { December } \\
9,2005\end{array}$ & $\begin{array}{c}\text { January } 6 \\
2006\end{array}$ & $\begin{array}{c}\text { January } \\
20,2006\end{array}$ & $\begin{array}{c}\text { January } 27, \\
206\end{array}$ & $\begin{array}{c}\text { February } \\
10,2006\end{array}$ & Mean \\
\hline & & & & & & $\mathrm{N}-\mathrm{NO}_{3}{ }^{-}$ & $\left(\mathrm{mg} \mathrm{L}^{-1}\right)$ & & & \\
\hline \multirow{2}{*}{1} & \multirow{2}{*}{1, tobacco } & 0.2 & $0^{*}$ & 0 & 4.3 & 0 & 10.3 & 9.8 & 14.2 & 5.5 \\
\hline & & 0.5 & 19.0 & 0 & 4.8 & 0 & 8.7 & 5.3 & 21.0 & 8.4 \\
\hline \multirow{2}{*}{1} & \multirow{2}{*}{2 , tobacco } & 0.2 & 11.9 & 0 & 0 & 9.8 & 11.8 & 11.8 & 28.1 & 10.5 \\
\hline & & 0.5 & 56.1 & 0 & 0 & 0 & 0 & 5.1 & 0 & 8.7 \\
\hline \multirow{2}{*}{1} & \multirow{2}{*}{3, tobacco } & 0.2 & 31.8 & 0 & 1.5 & 3.6 & 16.0 & 19 & 32.8 & 15.0 \\
\hline & & 0.5 & 28.9 & 0 & 1.6 & 0 & 13.8 & 15.1 & 0 & 8.5 \\
\hline \multirow{2}{*}{1} & \multirow{2}{*}{4 , tobacco } & 0.2 & 0 & 0 & 0 & 0 & 36.4 & 40.1 & 15.9 & 13.2 \\
\hline & & 0.5 & 79.9 & 37.6 & 2.0 & 4.4 & 19 & 24.2 & 18.5 & 26.5 \\
\hline \multirow{2}{*}{2} & \multirow{2}{*}{1 , tobacco } & 0.2 & 33.7 & 0 & 5.6 & 13.0 & 7.2 & 9.2 & 0 & 9.8 \\
\hline & & 0.5 & 0 & 0 & 0 & 0 & 0 & 21.5 & 0 & 3.1 \\
\hline \multirow{2}{*}{2} & \multirow{2}{*}{2, tobacco } & 0.2 & 43.7 & 0 & 0 & 0 & 0 & 0 & 0 & 6.2 \\
\hline & & 0.5 & 52.8 & 17.6 & 4.7 & 0 & 33.3 & 0 & 0 & 15.5 \\
\hline \multirow{2}{*}{2} & \multirow{2}{*}{4 , tobacco } & 0.2 & 158.4 & 4.8 & 4.1 & 0 & 0 & 0 & 23.8 & 27.3 \\
\hline & & 0.5 & 52.0 & 0 & 19.5 & 0 & 26.2 & 27 & 30.0 & 22.1 \\
\hline \multirow{2}{*}{3} & \multirow{2}{*}{1 , tobacco } & 0.2 & 0 & 0 & 0 & 0 & 14.5 & 17.6 & 0 & 4.6 \\
\hline & & 0.5 & 0 & 0 & 0 & 0 & 0 & 10.9 & 0 & 1.6 \\
\hline \multirow{2}{*}{3} & \multirow{2}{*}{2 , tobacco } & 0.2 & 71.2 & 0 & 3.2 & 0 & 5.1 & 14.3 & 16.4 & 15.7 \\
\hline & & 0.5 & 19.5 & 0 & 0 & 0 & 0 & 13.5 & 23.5 & 8.1 \\
\hline 4 & 1. tobacco & 0.2 & 17.1 & 6.4 & 2.3 & 2.9 & 8.3 & 1.2 & 1.0 & 5.6 \\
\hline & 19. & 0.5 & 0 & 7.0 & 4.2 & 0 & 29.8 & 31.6 & 2.3 & 10.7 \\
\hline 4 & 2 tobacco & 0.2 & 29.5 & 11.3 & 3.7 & 0 & 3.8 & 4.9 & 2.6 & 8.0 \\
\hline 1 & 2, & 0.5 & 39.5 & 0 & 3.3 & 0 & 6.5 & 7.8 & 1.0 & 8.3 \\
\hline 4 & 3 tobacco & 0.2 & 0.0 & 11.7 & 10.8 & 13.1 & 10.2 & 11.2 & 13.2 & 10.0 \\
\hline$T$ & 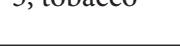 & 0.5 & 24.2 & 17.8 & 6.3 & 0 & 1.8 & 9.3 & 6.6 & 9.4 \\
\hline & Mean & & 32.1 & 4.8 & 3.4 & 2.0 & 10.9 & 12.9 & 10.5 & 10.9 \\
\hline 3 & 3 orassland & 0.2 & 7.3 & 0.9 & 0 & 0 & 0 & 0 & 0 & 1.2 \\
\hline & & 0.5 & 0.0 & 0 & 0 & 0 & 0 & 0 & 0 & 0.0 \\
\hline 3 & 4. grassland & 0.2 & 0.4 & 0 & 0.3 & 0 & 0 & 0.1 & 0 & 0.1 \\
\hline & & 0.5 & 0.0 & 1.6 & 1.1 & 4.8 & 4.9 & 3.1 & 3.5 & 2.7 \\
\hline & Mean & & 1.9 & 0.6 & 0.4 & 1.2 & 1.2 & 0.8 & 0.9 & 1.0 \\
\hline 2 & 3 , forest & 0.2 & 0 & 0 & 0 & 0 & 0 & 0 & 0 & 0.0 \\
\hline & & 0.5 & 39.1 & 0 & 24.8 & 0 & 0 & 1.3 & 0 & 9.3 \\
\hline 4 & 4 forest & 0.2 & 7.5 & 7.6 & 6.7 & 8.5 & 10.8 & 11.5 & 9.7 & 8.9 \\
\hline & & 0.5 & 8.4 & 9.3 & 9.2 & 10.0 & 9.8 & 10.9 & 11.0 & 9.8 \\
\hline 5 & 1 forest & 0.2 & 9.3 & 21.1 & 16.5 & 0 & 1.7 & 0 & 0 & 6.9 \\
\hline & $1,10100 \mathrm{~s}$ & 0.5 & 0 & 0 & 0 & 0 & 0 & 0 & 0 & 0.0 \\
\hline 5 & 2 forest & 0.2 & 4.3 & 27.6 & 14.1 & 0 & 2.5 & 0 & 1.0 & 7.1 \\
\hline & & 0.5 & 25.8 & 0 & 15.4 & 0 & 4.0 & 0.7 & 0 & 6.6 \\
\hline 5 & 3 forest & 0.2 & 8.1 & 2.8 & 0 & 0 & 2.0 & 0.7 & 1.0 & 2.1 \\
\hline & & 0.5 & 0 & 2.5 & 2.6 & 0 & 0 & 0.1 & 1.0 & 0.9 \\
\hline & Mean & & 10.3 & 7.1 & 8.9 & 1.9 & 3.1 & 2.5 & 2.4 & 5.7 \\
\hline
\end{tabular}

${ }^{*}$ The periods without data represent dates when it was not possible to collect the soil solution due to low soil moisture at these points. Position: 1: upper slope, 2: mid slope, 3: lower slope, and 4: plateau. 
TABLE 4: Orthogonal contrasts for nitrate concentrations in soil solutions between different positions in landscape, between collection times, and between land uses.

\begin{tabular}{|c|c|c|}
\hline \multirow{2}{*}{ Contrast } & \multicolumn{2}{|c|}{ Depth (m) } \\
\hline & 0.2 & 0.5 \\
\hline \multicolumn{3}{|c|}{ Positions in the landscape } \\
\hline $1 \times 2$ & ns & ns \\
\hline $1 \times 3$ & ns & ns \\
\hline $1 \times 4$ & $*$ & $* *$ \\
\hline \multicolumn{3}{|c|}{ Land use } \\
\hline Tobacco $\times$ grassland & ns & $*$ \\
\hline Tobacco $\times$ forest & ns & ns \\
\hline \multicolumn{3}{|c|}{ Collection periods } \\
\hline $1 \times 2$ & $*$ & $*$ \\
\hline $1 \times 3$ & * & * \\
\hline $1 \times 4$ & * & * \\
\hline $1 \times 5$ & * & * \\
\hline $1 \times 6$ & $*$ & $*$ \\
\hline $1 \times 7$ & $*$ & $*$ \\
\hline \multicolumn{3}{|c|}{$\begin{array}{l}{ }^{*} 5 \% \text { significant; }{ }^{* *} 1 \% \text { significant; ns: nonsignificant. Collection periods: } 1= \\
\text { November 10, } 2005 \text { (60 days after transplanting (DAT)); } 2 \text { = November } 28 \text {, } \\
2005 \text { (78 DAT); } 3=\text { December 09, } 2005 \text { (89 DAT); } 4=\text { January 06, } 2006 \text { (117 } \\
\text { DAT); } 5=\text { January 20, } 2006 \text { (131 DAT); } 6 \text { = January 27, } 2006 \text { (138 DAT); and } \\
7 \text { = February 10, } 2006 \text { (145 DAT). Position in the landscape: 1: upper slope, 2: } \\
\text { mid slope, 3: lower slope, and 4: plateau. }\end{array}$} \\
\hline
\end{tabular}

nitrogen. This "new organic matter" has higher nitrogen content, which helps in explaining high $\mathrm{N}_{-} \mathrm{NO}_{3}{ }^{-}$measured in revegetated forest and grassland (mean values of $7.2 \mathrm{mg} \mathrm{L}^{-1}$ ).

Nitrate concentration in soil solution from tobacco fields was higher than in natural environment but lower than values found immediately after tobacco transplanting when nitrate concentration rises above $100 \mathrm{mg} \mathrm{L}^{-1}$ [27]. Soil solution collected with lysimeters represents the fraction retained by soil after rainfall, since tension application is only possible under high soil moisture conditions [36]. Preferential flow via soil fissures and pores that are common in soils with coarse fractions [37] is ignored. The lysimeters were installed in sites where soil had favorable conditions and, thus, nitrate concentration data in soil solution presented herein may be underestimated, since soil hydraulic conditions are favorable to fast water drainage, increasing leaching losses.

Nitrate contents found in our study are comparable to concentrations observed for different crops. Oliveira et al. [38] observed loss of $76 \mathrm{~kg} \mathrm{ha}^{-1}$ due to leaching when $190 \mathrm{~kg} \mathrm{ha}^{-1}$ of nitrogen was applied in sugarcane. When total nitrogen applied was $803,1,607$, and $2,388 \mathrm{~kg} \mathrm{ha}^{-1}$ (sewage sludge), average $\mathrm{N}^{-\mathrm{NO}_{3}}{ }^{-}$contents in soil solution reached $29 \mathrm{mg} \mathrm{L}^{-1}, 39 \mathrm{mg} \mathrm{L}^{-1}$, and $85 \mathrm{mg} \mathrm{L}^{-1}$, respectively. The highest concentrations and losses occurred during the first samplings after fertilizer application.

Nitrate leached below root system has limited upward capillarity movement because of the large amount of coarse particles and macropores. Hence, little nitrate at $0.5 \mathrm{~m}$ is absorbed by tobacco crops, since rooting system is mainly located in the first $0.2 \mathrm{~m}$ layer. Besides, nitrate applied at

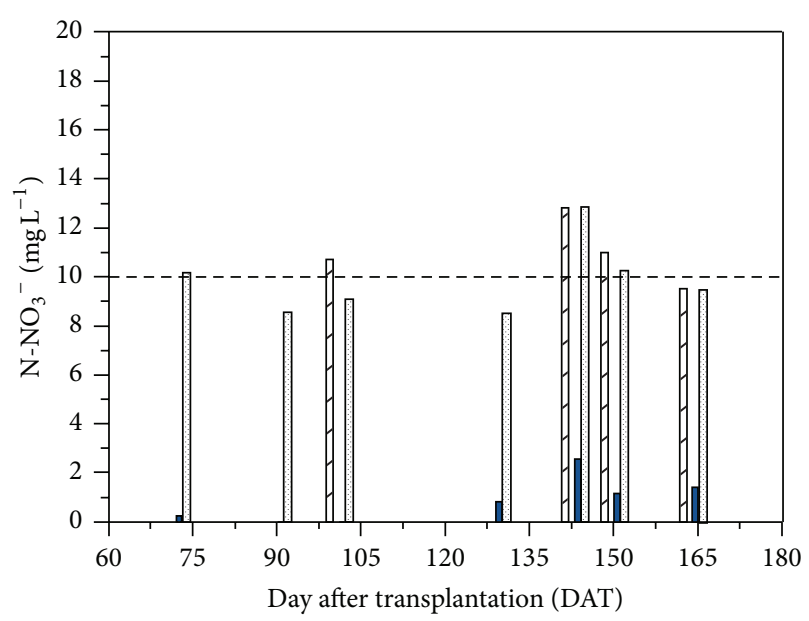

Water from the wells

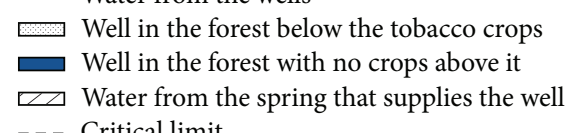

- - - Critical limit

FIGURE 4: Nitrate concentration in water from the sources used for domestic supply.

transplanting and at first topdressing is usually more susceptible to leaching due to plant's low nitrogen demand during this crop phase [9].

Conventional soil tillage maintains low cover during tobacco growth, exposes soil to erosion, and increases soil organic matter decomposition. Additionally, in tobacco crops, plant residues (stems and roots) are very low, inducing the use of greater quantities of mineral fertilizers [39].

Mean nitrogen dose applied in this watershed was $177 \mathrm{~kg} \mathrm{ha}^{-1}$. Considering that (i) mean leaf production was $2,114 \mathrm{~kg} \mathrm{ha}^{-1}$, (ii) mean root and stem dry mass was $2,358 \mathrm{~kg} \mathrm{ha}^{-1}$ [40], and (iii) average nitrogen content was $2.6 \%$ in leaves [41] and $1.1 \%$ in roots and stems [42], it can be estimated that $56 \mathrm{~kg} \mathrm{ha}^{-1}(32 \%)$ is exported with leaves and $26 \mathrm{~kg} \mathrm{ha}^{-1}(14 \%)$ remains in stems and roots. Therefore, $95 \mathrm{~kg} \mathrm{ha}^{-1}(54 \%)$ was not used by tobacco crop and is subject to migration into the aquatic systems, contaminating subsurface water resources [43], or is biotransformed.

Under optimal soil nutrient availability, tobacco crops absorb at most $70 \mathrm{~kg} \mathrm{ha}^{-1}$ of nitrogen to produce approximately $4,000 \mathrm{~kg} \mathrm{ha}^{-1}$ of dry leaf mass [9]. In Southern Brazil, 370,830 ha was cultivated to tobacco in 2010/2011 harvest [44], representing a loss of $3.52 \times 10^{7} \mathrm{~kg}$ of nitrogen. If all this nitrogen reached groundwater, it contaminated $3.52 \times 10^{12}$ liters of water to $10 \mathrm{mg} \mathrm{NO}_{3}^{-} \mathrm{L}^{-1}$ [15]. This shows the great invisible impact of tobacco farming on groundwater quality. It is worthy to mention that remaining soil nitrogen from previous crops and nitrogen supplied by soil organic matter mineralization were not considered in our estimations, since their contents are extremely low.

Landscape positions where groundwater is present in shallow soils and over solid rock are more prone to nitrate contamination, since leaching occurs by preferential flow in 
soil fissures. Besides, under these conditions denitrification is diminished by greater amount of oxygen present in drained water [14]. Nitrate contents in groundwater are major indicators of leaching and water contamination [21]. Preferential lateral flows may contaminate domestic water sources [45], as is the case in the Arroio Lino watershed.

Depth to water table is considered a major factor in water contamination [46]. In surface water table, infiltrated water reaches saturated zones faster, thus reducing the possibility of contaminants absorption or biodegradation [18]. To achieve efficient nitrate removal by denitrification, which is optimal in riparian zone, water flow must be slow [47]. Pionke and Lowrance [45] determined denitrification rate of $31 \mathrm{~kg} \mathrm{ha}^{-1}$ year $^{-1}$ for a riparian zone, while Nelson et al. [48] quantified nitrate removal capacity value of $120 \mathrm{~kg} \mathrm{ha}^{-1}$ year $^{-1}$. Under natural conditions, denitrification varies from 2 to $9 \%$ for well-drained soils with organic matter contents of less than $2 \%$ [43], as is the case in our watershed.

In our study, leached nitrate in soil profile had already reached surface water table as shown by the high nitrate level in water source below tobacco fields (Figure 4). According to the World Health Organization [14], methemoglobinemia may occur when nitrate ingestion varies from 0.09 to $45 \mathrm{mg} \mathrm{kg}^{-1}$ of the body weight. Nitrate concentration above $10 \mathrm{mg} \mathrm{L}^{-1}$ in water is considered critical by regulatory standards [15] and poses risk to human health, particularly to children under six months of age $[49,50]$.

Since nitrate is essentially originated from agriculture, as long as there are no nearby tobacco plantations, nitrate contents in water sources are usually below $3 \mathrm{mg} \mathrm{L}^{-1}$, representing noncontaminated groundwater by anthropic activities [14]. Forest nitrate filtering effect was not sufficient to reduce nitrate concentration below acceptable limits, probably due to high water flow, which diminishes water permanence time in the biologically active soil layer [20]. In general, a limiting factor in denitrification in aquifers is the availability of oxidable organic carbon [35], provided population of denitrifiers naturally occurs in groundwater [51].

In tobacco farms in Southern Brazil, percolation due to a coarse soil granulometry along with conventional soil tillage and low crop residues input are the main causes of nitrogen loss. Thus, protecting wells with a brickwork structure is ineffective in reducing water contamination by nitrate, and other alternatives must be sought to reduce the input of mineral fertilizers and to further improve management systems for soil organic nitrogen buildup. It is also important to seek adequate planning of soil use and wells location, to reduce environmental impact in these fragile environments.

Splitting nitrogen dose in tobacco crop is a good alternative to reducing water contamination by nitrate, to avoid having high nitrate concentration in the soil during periods of heavy rainfall. However, timing between the crop nitrogen needs and periods of intense rainfall is hard to be balanced and this enhances the loss of nitrogen in the system and water contamination. Additional alternatives are the adoption of no-tillage systems, use of cover crops and crop rotation, and promoting changes in land use in the watershed, by avoiding tobacco cultivation in areas with shallow and stony soils and leaving these areas under native vegetation or with grasses for grazing in silvopastoral systems. The restoration of riparian forests and the preservation of water wells are also needed in the watershed. This set of associated practices could minimize soil loss and contamination of surface and groundwater with $\mathrm{N}-\mathrm{NO}_{3}{ }^{-}$.

\section{Conclusion}

Soil physical and hydrological properties are highly variable at different landscape positions and soil depth. Coarse grain size, high porosity, and saturated hydraulic conductivity of the soil are conditions that favor nitrate losses by leaching. Concentration of nitrate in soil solution from tobacco fields is greater than in natural environment due to high amount and low efficiency of applied nitrogen fertilizers. Nitrogen leaching in tobacco fields contaminates water for human consumption. Large amounts of nitrogen fertilizers applied to tobacco and nitrate contamination of subsurface waters demonstrate the unsustainability of growing tobacco in small farming units on steeps slopes, with stony and shallow soils.

\section{Conflict of Interests}

The authors declare that there is no conflict of interests regarding the publication of this paper.

\section{References}

[1] A. G. Becker, B. S. Moraes, C. C. Menezes et al., "Pesticide contamination of water alters the metabolism of juvenile silver catfish, Rhamdia quelen," Ecotoxicology and Environmental Safety, vol. 72, no. 6, pp. 1734-1739, 2009.

[2] L. Sequinatto, J. M. Reichert, D. R. dos Santos, D. J. Reinert, and A. C. C. Copetti, "Occurrence of agrochemicals in surface waters of shallow soils and steep slopes cropped to tobacco," Química Nova, vol. 36, no. 6, pp. 768-772, 2013.

[3] N. B. Bonumá, C. H. G. Rossi, J. G. Arnold, J. M. Reichert, and E. M. C. D. Paiva, "Hydrology evaluation of the soil and water assessment tool considering measurement uncertainty for a small watershed in Southern Brazil," Applied Engineering in Agriculture, vol. 29, no. 2, pp. 189-200, 2013.

[4] G. Merten and J. P. G. Minella, "The expansion of Brazilian agriculture: soil erosion scenarios," International Soil and Water Conservation Research, vol. 1, pp. 37-48, 2013.

[5] N. B. Bonumá, C. G. Rossi, J. G. Arnold et al., "Simulating landscape sediment transport capacity by using a modified SWAT model," Journal of Environmental Quality, vol. 43, no. 1, pp. 55-66, 2014.

[6] E. J. Didoné, J. P. G. Minella, J. M. Reichert et al., "Impact of no-tillage agricultural systems on sediment yield in two large catchments in Southern Brazil," Journal of Soils and Sediments, vol. 14, no. 7, pp. 1287-1297, 2014.

[7] M. F. Rodrigues, J. M. Reichert, J. P. G. Minella et al., "Hydrosedimentology of nested subtropical watersheds with native and eucalyptus forests," Journal of Soils and Sediments, vol. 14, no. 7, pp. 1311-1324, 2014.

[8] J. P. G. Minella, G. H. Merten, D. E. Walling, and J. M. Reichert, "Changing sediment yield as an indicator of improved soil 
management practices in southern Brazil," Catena, vol. 79, no. 3, pp. 228-236, 2009.

[9] C. B. McCants and W. G. Woltz, "Growth and mineral nutrition of tobacco," Advances in Agronomy, vol. 19, no. C, pp. 211-265, 1967.

[10] B. Touraine, F. D. Vedele, and B. Forde, "Nitrate uptake and its regulation," in Plant Nitrogen, P. J. Lea and F. M. Gaudry, Eds., INRA editions, pp. 1-36, Springer, Berlin, Germany, 2001.

[11] C. T. MacKown and T. G. Sutton, "Recovery of fertilizer nitrogen applied to burley tobacco," Agronomy Journal, vol. 89, no. 2, pp. 183-189, 1997.

[12] M. I. Sifola and L. Postiglione, "The effect of nitrogen fertilization on nitrogen use efficiency of irrigated and non-irrigated tobacco (Nicotiana tabacum L.)," Plant and Soil, vol. 252, no. 2, pp. 313-323, 2003.

[13] SBCS-Sociedade Brasileira de Ciência do Solo, Manual de adubação e calagem para os Estados do Rio Grande do Sul e de Santa Catarina, Comissão de Química e Fertilidade do Solo, Núcleo Regional Sul, Porto Alegre, Brazil, 10 edition, 2004.

[14] World Health Organization (WHO), Rolling Revision of the WHO Guidelines for Drinking-Water Quality: Nitrates and Nitrites in Drinking-Water, 2004, http://www.who.int/water sanitation_health/dwq/chemicals/en/nitratesfull.pdf.

[15] CONAMA-Conselho Nacional do Meio Ambiente, Resolução $n^{\circ}$ 357, Ministério do Meio Ambiente, Brasília, Brasil, 2005.

[16] J. A. Moreno, Clima do Rio Grande do Sul, Secretaria da Agricultura, Diretoria de Terras e Colonização, Secção de Geografia, Porto Alegre, Brazil, 1961.

[17] G. H. Merten and J. P. Gomes Minella, "Impact on sediment yield due to the intensification of tobacco production in a catchment in Southern Brazil," Ciencia Rural, vol. 36, no. 2, pp. 669-672, 2006.

[18] E. Knox and D. W. Moody, "Influence of hydrology, soil properties and agricultural land use on nitrogen in groundwater," in Managing Nitrogen for Groundwater Quality and Farm Profitability, R. F. Follett, D. R. Keeney, and R. M. Cruse, Eds., pp. 19-56, Soil Science Society of America, Madison, Wis, USA, 1991.

[19] S. J. Smith and D. K. Cassel, "Estimating nitrate leaching in soil materials," in Managing Nitrogen for Groundwater Quality and Farm Profitability, R. F. Follett, D. R. Keeney, and R. M. Cruse, Eds., pp. 165-187, Soil Science Society of America, Madison, Wis, USA, 1991.

[20] J. H. Duff, A. P. Jackman, F. J. Triska, R. W. Sheibley, and R. J. Avanzino, "Nitrate retention in riparian ground water at natural and elevated nitrate levels in North Central Minnesota," Journal of Environmental Quality, vol. 36, no. 2, pp. 343-353, 2007.

[21] B. Lowery, R. C. Hartwig, D. E. Stoltenberg, K. J. Fermanich, and K. McSweeney, "Groundwater quality and crop-yield responses to tillage management on a Sparta sand," Soil and Tillage Research, vol. 48, no. 3, pp. 225-237, 1998.

[22] R. S. D. Dalmolin, F. A. Pedron, A. C. Azevedo, and A. Zago, Levantamento semidetalhado de solos da Microbacia do Arroio Lino-Município de Agudo-RS, FATEC/UFSM, Santa Maria, Brazil, 2004.

[23] D. L. Corwin, "Miscible solute transport: measurement of solute concentration using soil water extraction," in Methods of Soil Analysis, C. A. Black, Ed., pp. 1261-1266, American Sociey of Agronomy, Madison, Wis, USA, 1965.

[24] W. H. Hendershot and F. Courchesne, "Comparison of soil solution chemistry in zero tension and ceramic-cup tension lysimeters," Journal of Soil Science, vol. 42, no. 4, pp. 577-583, 1991.

[25] C. P. Webster, M. A. Shepherd, K. W. T. Goulding, and E. Lord, "Comparisons of methods for measuring the leaching of mineral nitrogen from arable land," Journal of Soil Science, vol. 44, no. 1, pp. 49-62, 1993.

[26] R. Poss, A. D. Noble, F. X. Dunin, and W. Reyenga, "Evaluation of ceramic cup samplers to measure nitrate leaching in the field," European Journal of Soil Science, vol. 46, no. 4, pp. 667-674, 1995.

[27] D. R. Kaiser, D. J. Reinert, J. M. Reichert, C. A. Streck, and A. Pellegrini, "Nitrate and ammonium in soil solution in different management systems for tobacco," Revista Brasileira de Ciencia do Solo, vol. 34, no. 2, pp. 379-387, 2010.

[28] D. L. Sparks, Methods of Soil Analysis. Part 3. Chemical Methods, Soil Science Society of America Book Series, No. 5, New York, NY, USA, 1996.

[29] SAS Institute, SAS/STAT Procedure Guide for Personal Computers, version 5, Statistical Analysis System Institute, Cary, NC, USA, 1991.

[30] T. J. Sauer and S. D. Logsdon, "Hydraulic and physical properties of stony soils in a small watershed," Soil Science Society of America Journal, vol. 66, no. 6, pp. 1947-1956, 2002.

[31] E. Epstein, W. J. Grant, and R. A. Struchtemeyer, "Effects of stones on runoff, erosion and soil moisture," Soil Science Society of America Journal, vol. 30, no. 5, pp. 638-640, 1966.

[32] J. C. Fiès, N. de Louvigny, and A. Chanzy, "The role of stones in soil water retention," European Journal of Soil Science, vol. 53, no. 1, pp. 95-104, 2002.

[33] M. G. B. F. Mesquita and S. O. Moraes, "A dependência entre a condutividade hidráulica saturada e atributos físicos do solo," Ciência Rural, vol. 34, no. 3, pp. 963-969, 2004.

[34] U. K. Mandal, K. V. Rao, P. K. Mishra et al., "Soil infiltration, runoff and sediment yield from a shallow soil with varied stone cover and intensity of rain," European Journal of Soil Science, vol. 56, no. 4, pp. 435-443, 2005.

[35] T. R. Steinheimer, K. D. Scoggin, and L. A. Kramer, "Agricultural chemical movement through a field-size watershed in Iowa: subsurface hydrology and distribution of nitrate in groundwater," Environmental Science and Technology, vol. 32, no. 8, pp. 1039-1047, 1998.

[36] J. Grossmann and P. Udluft, "The extraction of soil water by the suction-cup method: a review," Journal of Soil Science, vol. 42, no. 1, pp. 83-93, 1991.

[37] E. L. McCoy, C. W. Boast, R. C. Stehouwer, and E. J. Kladivko, "Macropore hydraulics: taking a sledgehammer to classical theory," in Soil Processes and Water Quality, R. Lal and B. A. Stewart, Eds., Advances in Soil Science, pp. 303-348, Lewis Publishers, Ann Arbor, Mish, USA, 1994.

[38] F. C. Oliveira, M. E. Mattiazzo, C. R. Marciano, and S. O. Moraes, "Lixiviação de nitrato em um Latossolo Amarelo distrófico tratado com lodo de esgoto e cultivado com cana-de-açúcar," Scientia Agricola, vol. 58, no. 1, pp. 171-180, 2001.

[39] G. A. Peterson and J. F. Power, "Soil, crop and water management," in Managing Nitrogen for Groundwater Quality and Farm Profitability, R. F. Follett, D. R. Keeney, and R. M. Cruse, Eds., pp. 189-198, Soil Science Society of America, Madison, Wis, USA, 1991.

[40] A. Pellegrini, Sistemas de cultivo da cultura do fumo com ênfase às práticas de manejo e conservação do solo [M.S. dissertation], Universidade Federal de Santa Maria, Santa Maria, Brazil, 2006. 
[41] D. S. Rheinheimer, H. R. Bartz, J. Kaminski, and S. A. Willani, "Substituição do sulfato de potássio por cloreto na mistura de fertilizantes para a cultura do fumo," Agronomia Sulriograndense, vol. 27, pp. 35-46, 1991.

[42] E. B. Whitty and R. N. Gallaher, Supplemental Nitrogen Fertilizer for No-Till Tobacco Following Simulated Excessive Rainfall, Institute of Food and Agricultural Science, Gainesville, Fla, USA, 1995, http://www.ag.auburn.edu/auxiliary/nsdl/scasc/ Proceedings/1995/Whitty.pdf.

[43] J. J. Meisinger and G. W. Randall, "Estimating nitrogen budgets for soil-crop systems," in Managing Nitrogen for Groundwater Quality and Farm Profitability, R. F. Follett, D. R. Keeney, and R. M. Cruse, Eds., pp. 85-124, Soil Science Society of America, Madison, Wis, USA, 1991.

[44] AFUBRA, Associação dos Fumicultores do Brasil, December 2010, http://www.afubra.com.br.

[45] H. B. Pionke and R. R. Lowrance, "Fate of nitrate in subsurface drainage waters," in Managing Nitrogen for Groundwater Quality and Farm Profitability, R. F. Follett, D. R. Keeney, and R. M. Cruse, Eds., pp. 237-255, Soil Science Society of America, Madison, Wis, USA, 1991.

[46] P. J. Squillace, J. C. Scott, M. J. Moran, B. T. Nolan, and D. W. Kolpin, "VOCs, pesticides, nitrate, and their mixtures in groundwater used for drinking water in the United States," Environmental Science and Technology, vol. 36, no. 9, pp. 19231930, 2002.

[47] J. C. Rutherford and M. L. Nguyen, "Nitrate removal in riparian wetlands: interactions between surface flow and soils," Journal of Environmental Quality, vol. 33, no. 3, pp. 1133-1143, 2004.

[48] W. M. Nelson, A. J. Gold, and P. M. Groffman, "Spatial and temporal variation in groundwater nitrate removal in a riparian forest," Journal of Environmental Quality, vol. 24, no. 4, pp. 691699, 1995.

[49] E. V. S. Prakasa Rao and K. Puttanna, "Nitrates, agriculture and environment," Current Science, vol. 79, no. 9, pp. 1163-1168, 2000.

[50] T. M. Addiscott and N. Benjamin, "Nitrate and human health," Soil Use and Management, vol. 20, no. 2, pp. 98-104, 2004.

[51] D. Haag and M. Kaupenjohann, "Landscape fate of nitrate fluxes and emissions in Central Europe: a critical review of concepts, data, and models for transport and retention," Agriculture, Ecosystems and Environment, vol. 86, no. 1, pp. 1-21, 2001. 

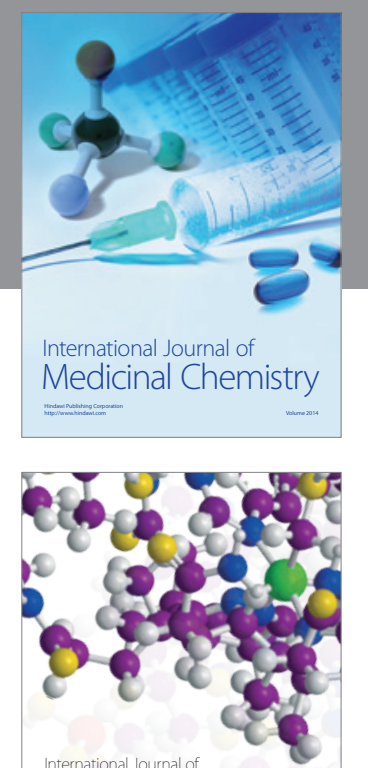

\section{Carbohydrate} Chemistry

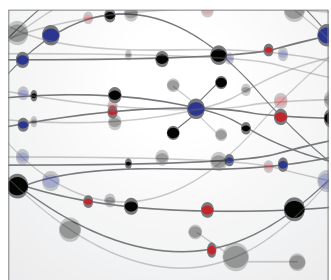

The Scientific World Journal
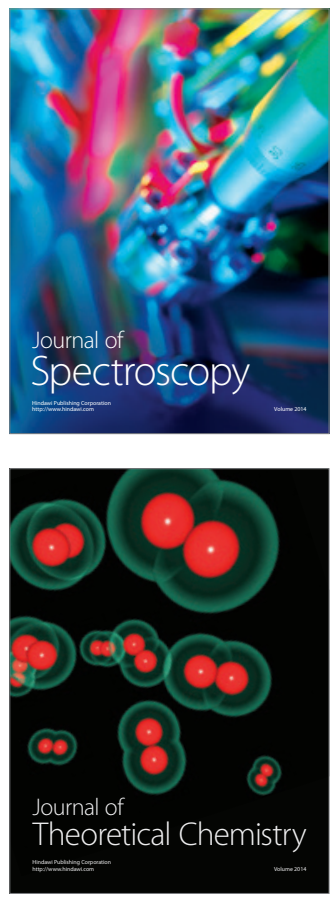
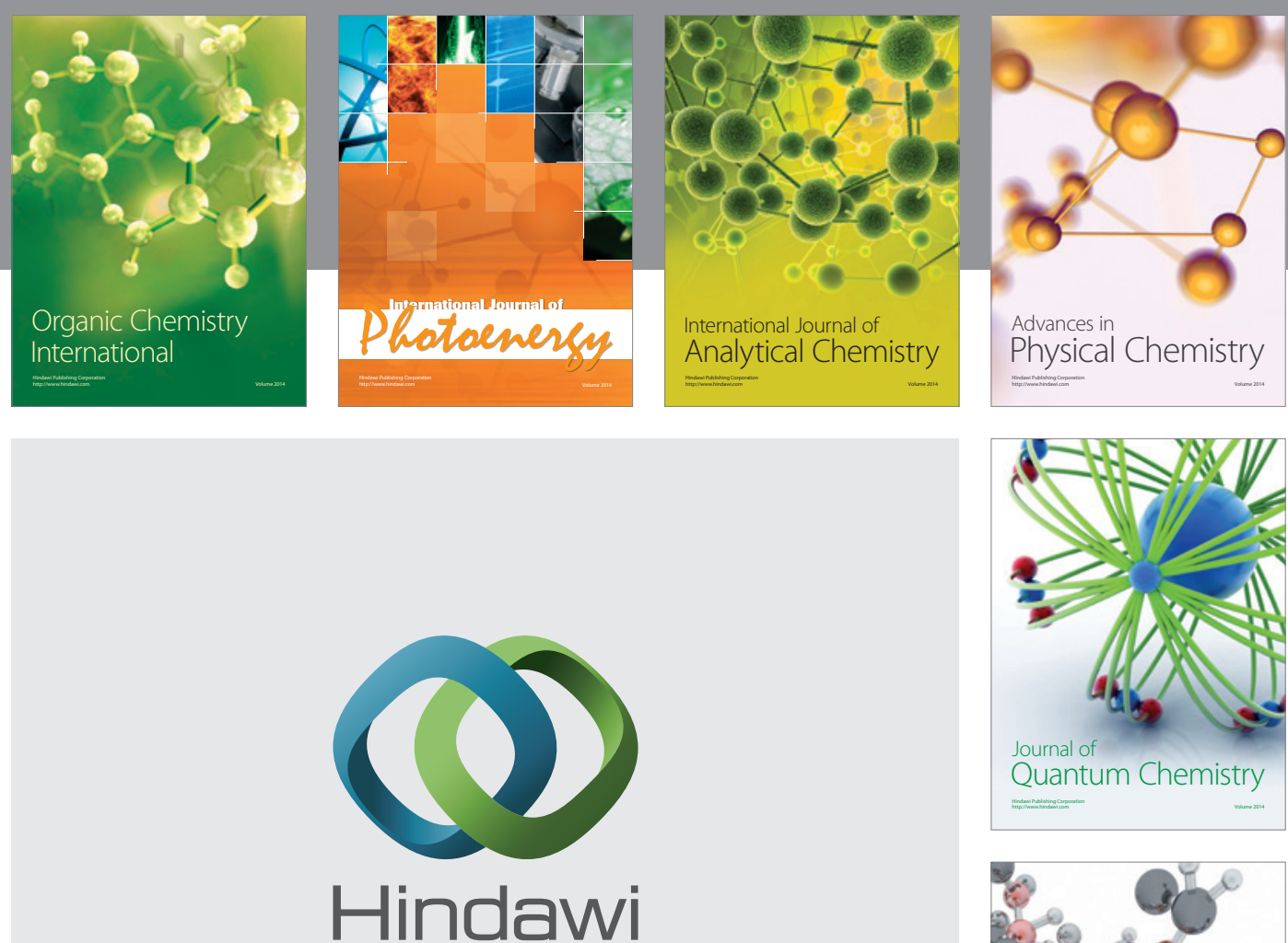

Submit your manuscripts at

http://www.hindawi.com

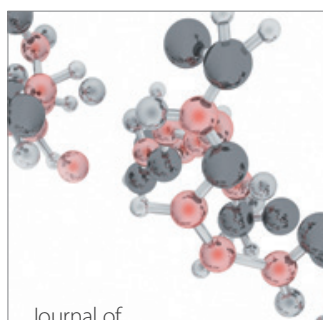

Analytical Methods

in Chemistry

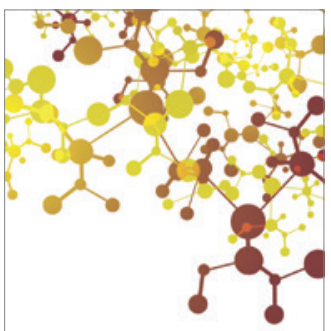

Journal of

Applied Chemistry

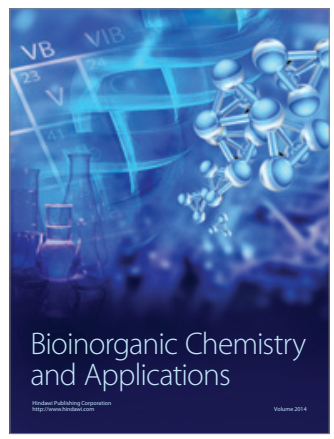

Inorganic Chemistry
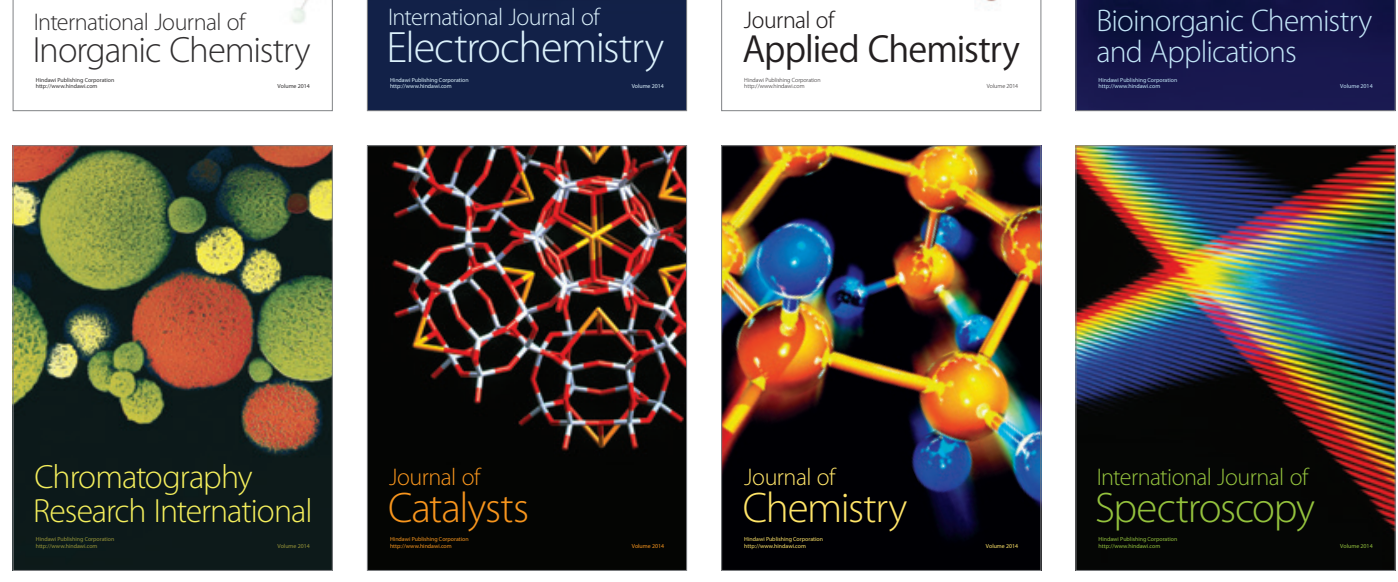